\title{
Evaluation of a point-of-care tuberculosis test-and-treat algorithm on early mortality in people with HIV accessing antiretroviral therapy (TB Fast Track study): study protocol for a cluster randomised controlled trial
}

Katherine L Fielding ${ }^{1 *}$, Salome Charalambous ${ }^{2}$, Christopher J Hoffmann ${ }^{3}$, Suzanne Johnson ${ }^{4}$, Mpho Tlali ${ }^{2}$, Susan E Dorman ${ }^{3}$, Anna Vassall ${ }^{5}$, Gavin J Churchyard ${ }^{1,2}$ and Alison D Grant ${ }^{6}$

\begin{abstract}
Background: Early mortality for HIV-positive people starting antiretroviral therapy (ART) remains high in resource-limited settings, with tuberculosis the most important cause. Existing rapid diagnostic tests for tuberculosis lack sensitivity among HIV-positive people, and consequently, tuberculosis treatment is either delayed or started empirically (without bacteriological confirmation). We developed a management algorithm for ambulatory HIV-positive people, based on body mass index and point-of-care tests for haemoglobin and urine lipoarabinomannan (LAM), to identify those at high risk of tuberculosis and mortality. We designed a clinical trial to test whether implementation of this algorithm reduces six-month mortality among HIV-positive people with advanced immunosuppression.

Methods/design: The TB Fast Track study is an open, pragmatic, cluster randomised superiority trial, with 24 primary health clinics randomised to implement the intervention or standard of care. Adults (aged $\geq 18$ years) with a CD4 count of 150 cells/ $\mu \mathrm{L}$ or less, who have not received any tuberculosis treatment in the last three months, or ART in the last six months, are eligible. In intervention clinics, the study algorithm is used to classify individuals as at high, medium or low probability of tuberculosis. Those classified as high probability start tuberculosis treatment immediately, followed by ART after two weeks. Medium-probability patients follow the South African guidelines for test-negative tuberculosis and are reviewed within a week, to be re-categorised as low or high probability. Low-probability patients start ART as soon as possible. The primary outcome is all-cause mortality at six months. Secondary outcomes include severe morbidity, time to ART start and cost-effectiveness.

Discussion: This trial will test whether a primary care-friendly management algorithm will enable nurses to identify HIV-positive patients at the highest risk of tuberculosis, to facilitate prompt treatment and reduce early mortality. There remains an urgent need for better diagnostic tests for tuberculosis, especially for people with advanced HIV disease, which may render empirical treatment unnecessary.
\end{abstract}

Trial registration: This trial was registered with Current Controlled Trials (identifier: ISRCTN35344604) on 12 September 2012.

Keywords: Tuberculosis, HIV infections, Pragmatic clinic trials, Mortality, Treatment

\footnotetext{
* Correspondence: katherine.fielding@lshtm.ac.uk

'Department of Infectious Disease Epidemiology, London School of Hygiene

\& Tropical Medicine, Keppel Street, London WC1E 7HT, UK

Full list of author information is available at the end of the article
} 


\section{Background}

\section{Background and rationale}

Early mortality among HIV-positive people starting antiretroviral therapy (ART) remains higher in resourceconstrained settings compared with industrialised countries [1]. Tuberculosis is the most important cause of death among people with HIV worldwide [2], and limited data suggest that this remains true of people starting ART [3]. However, the diagnosis of tuberculosis is difficult and may be missed altogether, particularly among people with low CD4 counts, who are less likely to be diagnosed using sputum-based tests because of lower mycobacterial concentration or inability to produce sputum, and may have atypical appearances on chest radiography. Sputum mycobacterial culture is the gold standard diagnostic test for tuberculosis, but is not routinely available in many resource-constrained settings. Where culture is available, the result may take up to six weeks, which may delay ART initiation [4].

New diagnostic tests for tuberculosis are increasingly available. In South Africa, Xpert MTB/RIF has replaced sputum microscopy as the first diagnostic test for tuberculosis [5]. With an instrument turnaround time of under two hours, Xpert MTB/RIF has the potential to provide point-of-care testing with in-session results, but the instrument requirements, its moderate complexity $[6,7]$ and high cost mean that it is unlikely to be widely implemented at primary care level in resourceconstrained settings. Xpert MTB/RIF has better sensitivity than microscopy, but is less sensitive than sputum culture on liquid media [8]. Further, in two trials comparing Xpert MTB/RIF to sputum smear microscopy, Xpert did not improve patient-relevant outcomes. In the XTEND trial, nested within national roll-out of Xpert MTB/RIF in South Africa, placed in off-site laboratories, there was no reduction in mortality among people being investigated for tuberculosis [9]. In the TB-NEAT trial Xpert MTB/RIF was deployed in primary care clinics to give a same-day result, with no resulting reduction in morbidity [10]. In both trials, a likely explanation was that many people were treated empirically (that is, without microbiological confirmation) for tuberculosis, and that Xpert MTB/RIF provided microbiological confirmation for some cases which would otherwise have been treated empirically, but found few additional cases.

Empirical treatment of tuberculosis is common, but often requires a physician's decision. Many primary care clinics are run by nurses with physicians attending only occasionally, if at all, and in such settings empirical treatment may be delayed, or not initiated. We sought to develop an algorithm which would enable nurses in primary care settings to identify, among HIV-positive people with advanced immunosuppression who are at high risk of death, those at the highest risk of tuberculosis, in order to start empirical tuberculosis treatment promptly, followed by ART.

A promising candidate component of this algorithm was an assay for lipoarabinomannan (LAM), a mycobacterial cell-wall component which can be detected in urine among patients with tuberculosis. A lateral flow assay for LAM is commercially available as a point-of-care test, giving a result in 25 minutes at relatively low cost which, with no requirement for sample processing, has potential for use in primary care clinics. The test's sensitivity is too low to be useful for HIV-negative people. Even among HIVpositive people, sensitivity is inadequate, except among those with very low CD4 counts [11]. We therefore selected two additional markers for our algorithm: body mass index (BMI) and haemoglobin. Among people with HIV, low BMI and low haemoglobin levels are strongly associated with early mortality, and also with active tuberculosis [12-17]. Both BMI and haemoglobin can be measured by a nurse in a primary care setting with in-session results. Additional analysis of data from a study evaluating LAM among hospitalised patients in South Africa with signs or symptoms of tuberculosis [18] helped refine the algorithm.

We therefore decided to evaluate an algorithm combining haemoglobin, BMI and urine LAM lateral flow assay with symptom screening for tuberculosis, to classify study patients as having high, medium or low probability of active tuberculosis (Figure 1), and accordingly start presumptive tuberculosis treatment, ART or both, with minimal delay.

\section{Rationale for a randomised controlled trial}

Our overall aim was to enable the rapid initiation of tuberculosis treatment among high-risk people, and thus reduce early mortality due to tuberculosis. Rapid initiation of tuberculosis treatment should also facilitate early initiation of ART, thus reducing early mortality due to a wide range of conditions associated with advanced HIV disease. Other potential advantages include a lower risk of morbidity associated with immune reconstitution syndrome due to tuberculosis, reduced morbidity due to rifampicin-sensitive bacterial infections and effective treatment for latent tuberculosis infection.

However, a strategy promoting rapid initiation of tuberculosis treatment without bacteriological confirmation has potential disadvantages. For patients, some will receive tuberculosis treatment when they do not, in reality, have active tuberculosis, with attendant risks of adverse effects and drug interactions. Some patients with unidentified drug-resistant tuberculosis may be inappropriately treated with a regimen appropriate only for drug-sensitive tuberculosis. The focus of the algorithm on tuberculosis could also delay investigation and treatment for alternative comorbidities. For the health system, disadvantages include 


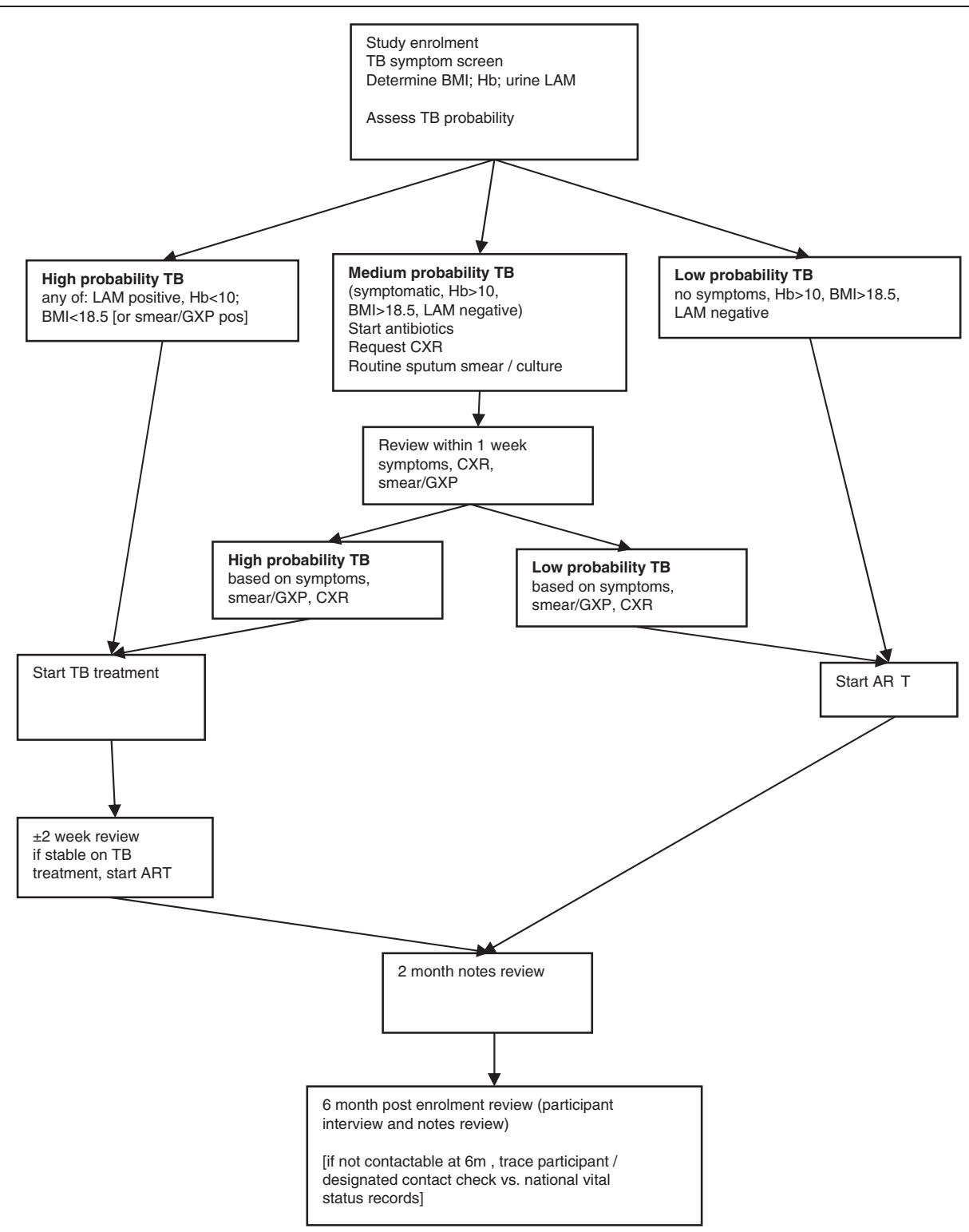

Figure 1 TB Fast Track management algorithm. ART: antiretroviral therapy; BMI: body mass index; CXR: chest radiograph; GXP: Xpert MTB/RIF; Hb: haemoglobin; LAM: lipoarabinomannan (urine assay for tuberculosis); TB: tuberculosis.

a higher caseload of patients requiring tuberculosis treatment, and an apparent increase in tuberculosis case notification rates. Given uncertainties concerning whether the expected benefits of our novel management algorithm would outweigh the risks, we propose to conduct a clinical trial.

\section{Rationale for a cluster randomised trial}

This trial could broadly be considered a health service delivery trial; for such trials, there is debate about whether randomisation should be at the individual or cluster level $[19,20]$. Advantages of cluster randomisation include minimising contamination, in that where patients in the same clinic are randomised to different arms, elements of the intervention may be implemented among patients allocated to the control arm. Other advantages include logistical convenience and approximating more closely how the intervention would be delivered in routine practice. Disadvantages include a larger sample size for the same power and effect size, complexities of revising sample size calculations if necessary, challenges in controlling for confounding and standardising delivery of the intervention. We decided on randomisation at clinic level primarily because we anticipated that contamination would be likely if individuals in one clinic could be randomised to either intervention or control arm. 


\section{Hypothesis}

We hypothesise that, for adults with HIV with a CD4 count of 150 cells/ $\mu \mathrm{L}$ or less who are not taking ART, a care pathway using point-of-care tests to rapidly identify individuals at high risk of tuberculosis and ensure initiation of tuberculosis treatment followed by ART, will reduce mortality within the six months following enrolment.

\section{Methods/design}

\section{Study design}

The study is an open, pragmatic two-arm cluster randomised superiority trial, with primary health clinics as the unit of randomisation. The intervention is integrated into routine clinic activities as far as possible: study staff implement the algorithm, and all other aspects of care for the patient are delivered by clinic staff.

\section{Intervention arm - implementation of the algorithm}

The study nurse completes a questionnaire with consenting, eligible patients to determine tuberculosis symptoms and BMI, and performs point-of-care tests for haemoglobin concentration and urine LAM (Determine ${ }^{\text {TM }}$ TB LAM Ag, Alere, Waltham Massachusetts, United States). Patients are then classified as having high, medium or low probability of active tuberculosis (Figure 1).

Participants are classified as at a high probability of tuberculosis if they have: (i) a positive urine LAM result (defined as any positive band on the test strip, graded against the manufacturer's pre-January 2014 reference card), (ii) a haemoglobin concentration $<10 \mathrm{~g} / \mathrm{dL}$ or (iii) a BMI $<18.5 \mathrm{~kg} / \mathrm{m}^{2}$ (along with any patient with a previous sputum smear or positive Xpert MTB/RIF test [Cepheid, Sunnyvale, CA, USA] result). They are started on tuberculosis treatment immediately, followed by ART as soon as possible.

Participants classified as at a low probability of tuberculosis, defined as no symptoms suggestive of tuberculosis, a negative LAM result, a haemoglobin concentration $\geq 10 \mathrm{~g} /$ $\mathrm{dL}$ and a $\mathrm{BMI} \geq 18.5 \mathrm{~kg} / \mathrm{m}^{2}$ (and not known to be sputum smear or Xpert MTB/RIF positive) are started on ART (without tuberculosis treatment) as soon as possible.

Participants classified as at a medium probability of tuberculosis, that is, reporting any symptom suggestive tuberculosis but not satisfying the criteria for high probability, are managed in accordance with national guidelines for test-negative tuberculosis: given broad spectrum (non-quinolone) antibiotics if indicated, an additional routine sputum sample is taken for smear and mycobacterial culture in line with routine practice, and a chest radiography is arranged if not recently performed, with review within one week wherever possible. At review, they are classified as high or low probability, based on symptom review, response to antibiotic treatment and investigation results.
Patients with specific symptoms suggesting diagnoses other than tuberculosis are managed in accordance with national guidelines. For study participants who start tuberculosis treatment, an efavirenz-based ART regimen is used, to be compatible with tuberculosis treatment. Any patients unable or unwilling to take this ART regimen are excluded from the trial. Outside this situation, patients can take any ART regimen consistent with South African ART guidelines.

In order to have a reference 'gold standard' against which to assess the study algorithm, a sputum specimen is also collected at enrolment for smear, culture, organism identification and drug susceptibly testing at a research laboratory. Results are not available at the time of enrolment, and so do not contribute to the initial assignment of probability of tuberculosis.

\section{Standard of care}

Study staff recruit eligible, consenting patients and complete a short questionnaire, measure height and weight (for systematic measurement of BMI for analysis purposes). Further assessment for tuberculosis and preparation for ART is undertaken by clinic staff according to routine practice following national guidelines. In both arms of the study, dried blood spots and a urine sample are collected and stored. Intended tests include cryptococcal antigen and culture of urine for mycobacteria.

\section{Selection of study clinics}

The study is conducted in primary care clinics and community health centres in a mixture of urban and rural settings in Gauteng, North West and Limpopo provinces, South Africa. Exclusion criteria include having a laboratory doing sputum smear microscopy and Xpert $\mathrm{MTB} / \mathrm{RIF}$ tests onsite, with same day results. There was a deliberate decision to include rural sites on the basis that the intervention is particularly relevant to patients in rural settings, where long distances to clinics may be a barrier to speedy initiation of ART, particularly if multiple visits are needed.

\section{Study population}

The study population are HIV-positive adults ( $\geq 18$ years) with a CD4 count of 150 cells/ $\mu \mathrm{L}$ or less, who are willing to start ART. The trial aims to be as generalisable as possible so exclusion criteria are few and include, among others, patients currently on tuberculosis treatment, who have completed tuberculosis treatment in last three months or ART in the last six months, those with a contraindication to efavirenz and those who are too sick to be managed in ambulatory care. We chose a CD4 count threshold of 150 cells/ $\mu \mathrm{L}$ or less primarily because of the high risk of mortality in this group. A more standard 
CD4 count cutoff of under 200 cells $/ \mu \mathrm{L}$ would have made the study more widely applicable, though at a cost of having a larger sample size due to a lower mortality rate among ART initiators with a CD4 count between 150 and 200 cells $/ \mu \mathrm{L}$.

\section{Trial outcomes}

The primary outcome is all-cause mortality measured over a six-month period following enrolment. Secondary outcomes are (i) duration of hospital admission in the first six months after enrolment, (ii) time from enrolment to ART start, (iii) proportion of patients retained in HIV care and (iv) serious and severe adverse events in specified categories as defined by the study (particularly hepatotoxicity, hypersensitivity, peripheral neuropathy and nephrotoxicity). The study has also defined economic outcomes relating to measuring cost-effectiveness which include total diagnostic cost of the study cohort in intervention and control arms, as well as incremental diagnostic, treatment and per disability-adjusted life year (DALY) costs of the intervention compared to control from a provider perspective, over the six-month followup period.

Vital status at six months from enrolment will be ascertained from patient interview and medical records, and supplemented with information from the participant's nominated next of kin for those lost to follow-up, and the South African mortality registration for participants with a South African identification number. If necessary, a trial endpoints committee will be convened, masked to study arm, to assign death endpoints where there are discrepant dates of death. Secondary outcomes will be measured using a combination of data from patient interview and medical records, abstracted at two and six months from enrolment. A substudy among study participants who die is being conducted, using needle and verbal autopsy methods to ascertain causes of deaths.

\section{Economic evaluation}

An economic evaluation is being conducted from a provider perspective. The primary outcome for the economic evaluation is incremental cost per death averted within the trial period. An additional analysis will be conducted to estimate incremental cost per DALY averted. The costs of the intervention are being collected throughout the trial period, with care taken to exclude any research-related costs. A bottom-up costing approach will be used to measure the costs associated with implementation of the point-of-care tests at the clinic level (cost per person assessed and costs for different components of the algorithm). Provider costs for all services in the patient pathway are being collected from a sample of six clinics, purposively selected based on the ratio between staffing levels and the number of clinic attendees. Patient-level data on health service use are being collected using the general trial instruments (follow-up visits) and case note data. Should the intervention be found cost-effective, a budget impact analysis will be conducted to estimate the costs of national scale-up.

\section{Sample size considerations}

Data from the TB and HIV Prevention, Care and Treatment programme of The Aurum Institute helped inform the sample size calculation for the primary outcome. Mortality in the first six months after ART initiation among those with a CD4 count of under 150 cells $/ \mu \mathrm{L}$ was $24 / 100$ person-years $[17,21,22]$. For the sample size calculation, this was considered a minimum estimate, given that ascertainment of deaths did not include a search of national death registrations for some of these studies, and mortality prior to ART exceeds mortality during ART. Given our study is designed to enrol patients prior to ART start we used a mortality estimate of $25 / 100$ person-years in the standard of care arm. The sample size calculation took into account the clustered design [19] through the coefficient of variation, which we assumed to be between 0.2 and 0.25 . We had no prestudy clinic-level mortality data to inform coefficient of variation between clinics, but given the study population were patients pre-ART, all with CD4 counts of 150 cells/ $\mu \mathrm{L}$ or less, we assumed a coefficient of variation no greater than 0.25 was reasonable.

Tuberculosis is identified at autopsy in 21 to $54 \%$ of HIV-positive adults in sub-Saharan Africa [2]. Given the very high prevalence of tuberculosis in South Africa [3], we assumed that $60 \%$ of early deaths in our study population would be attributable to tuberculosis, and that, through earlier initiation of tuberculosis treatment, $66 \%$ of these could be averted. Using data from the TB and HIV Prevention, Care and Treatment programme of The Aurum Institute, among individuals with a CD4 count between 100 and 150 cells $/ \mu \mathrm{L}$, a two-month delay in ART initiation resulted in a $150 \%$ increase in mortality by six months; from $7 \%$ with immediate ART initiation to $18 \%$ if there was a two-month delay [22]. There must be some overlap of preventable deaths between these two effects, which we could not quantify precisely. Overall, we assumed that the intervention had the potential to result in a $40 \%$ reduction in mortality by six months after study enrolment.

Assuming 10 clinics per arm, 175 patients per clinic, 5\% individuals whose vital status could not be ascertained at six months, estimated mortality of $25 / 100$ person-years in the standard of care arm and coefficients of variation of 0.2 and 0.25 , there would be $91 \%$ and $85 \%$ power to assess a $40 \%$ reduction in mortality, respectively. If the coefficient 
of variation is 0.2 , there would be $81 \%$ power to assess a $35 \%$ reduction in mortality.

After enrolling participants for six months we established that for some clinics, the target of 175 patients per clinic was unlikely to be achieved, and so the sample size calculations were revised. Assuming a harmonic mean of 109 patients per clinic, by randomising an additional four clinics, the study maintained similar power and effect sizes as with the original calculation.

\section{Randomisation}

The initial randomisation of 20 clinics was based on restriction, common in cluster randomised trials with a small number of clusters [19], and based on having reasonable balance, separately, for mean CD4 count, periurban/rural location of the clinic and the total number of new ART initiations per month. Using these criteria, $11,160(6.0 \%)$ of the 184,756 possible allocations for randomising 20 clinics to two arms (ratio of 1:1) were identified. The validity of this restriction was checked for each allocation by comparing the number of times a cluster appeared in the same arm with each other cluster. There was no extreme imbalance and this restriction was assumed to be valid. The randomisation was organised by a statistician. In a public ceremony with study clinics represented by clinic managers and other HIV and TB programme staff, one allocation was chosen at random. Due to the revision in sample size, a second public randomisation took place 14 months later, where four additional clinics were randomised in a ratio 1:1 to the intervention or standard of care arm.

\section{Data collection and management}

Case report forms were piloted in 10 clinics prior to the study start. Before a clinic formally initiated enrolment, the enrolment, one-week and two-week case report forms were tested in around 10 patients.

Written informed consent is obtained from all participants using information sheets available in relevant languages, with the assistance of a translator where necessary, using standard consent forms. Participants unable to read or write are asked to make a mark or thumbprint in the presence of a witness. Following enrolment, participants in the two arms are seen in accordance with the visit schedule summarised in Table 1. Study staff complete case report forms on carbonated paper in duplicate, and store forms at site in locked filing cabinets, with access to these records restricted to specified study team members. Case report forms are identified using the participant's study number only, with locator information stored separately.

Participant retention is crucial for this study, in particular for the measurement of the primary outcome. At enrolment in both arms, participants are asked to give locator information, including next of kin and their
South African identification number, as described previously. Participants are also asked to provide their mobile telephone number, and are called by study staff at one week, and one, two, four and six months to reconfirm contact details. Participants are encouraged to notify study staff of any relevant changes in their circumstances, such as moving away from the locality.

A quality control system is in place to monitor $100 \%$ of the consent forms and enrolment case report forms, and $10 \%$ of follow-up case report forms. More frequent monitoring of a site is initiated if problems are identified. Every week, across all 24 sites, the bottom copies of completed forms are sent to a central office for data entry in a password-protected, user-limited sequel database, with range checks on fields where appropriate. Queries based on data in the database are generated every one to two months and sent to sites for resolution. Data in the database from all case report forms from a random sample of $10 \%$ of study participants are verified. In addition, data on critical fields, primarily used for measurement of study endpoints, are verified in the database for $100 \%$ of study participants. Locator data are stored separately from the main database.

\section{Statistical analysis}

Analyses will use methods appropriate for the cluster randomised trial design, giving each cluster equal weight. Quantitative outcomes will be summarised as the mean for each cluster and the difference of means for the intervention versus standard of care arm. For binary outcomes or rate outcomes, the overall risk (rate) for each cluster will be calculated, as well as the ratio for the intervention versus standard of care arm. Given the overall randomisation was conducted using two strata (20 and four clinics in each stratum), the standard error for each effect measure will take into account stratified randomisation. An adjusted analysis will be conducted if, after visual inspection, imbalance by study arm in patient- or clinic-level factors is observed, to help reduce variability across clinics with respect to primary and secondary outcomes.

Subgroup analyses will be conducted for the primary outcome for baseline CD4 count $(<50$ or $\geq 50$ cells $/ \mu \mathrm{L})$, previous tuberculosis history (from self-report; no previous or previous tuberculosis), baseline BMI ( $<18.5$ or $\geq 18.5 \mathrm{~kg} /$ $\left.\mathrm{m}^{2}\right)$ and baseline haemoglobin $(<8$ or $\geq 8 \mathrm{~g} / \mathrm{dL})$. A statistical analysis plan documents the analysis of all trial outcomes.

\section{Ethics and dissemination}

The trial has approval from the Research Ethics Committees of the University of Witwatersrand (approval number: R14/49 M111177); the London School of Hygiene and Tropical Medicine, United Kingdom (approval number: 6099) and the Provincial Research Committees 


\begin{tabular}{|c|c|c|c|c|c|c|c|c|c|c|c|c|c|c|}
\hline & Enrolment & 1 week (or less) & 2 weeks & 1 month & 2 months & 4 months & 6 months & Enrolment & 1 week (or less) & 2 weeks & 1 month & 2 months & 4 months & 6 month \\
\hline Informed consent & $\sqrt{ }$ & & & & & & & $\sqrt{ }$ & & & & & & \\
\hline Locator information & $\sqrt{ }$ & & & & & & & $\sqrt{ }$ & & & & & & \\
\hline Baseline questionnaire & $\sqrt{ }$ & & & & & & & $\sqrt{ }$ & & & & & & \\
\hline TB symptom screen & $\sqrt{ }$ & $\sqrt{ }^{a}$ & & & & & & $\sqrt{ }$ & & & & & & \\
\hline Body mass index & $\sqrt{ }$ & & & & & & & $\sqrt{ }$ & & & & & & \\
\hline \multicolumn{15}{|l|}{ Lab tests } \\
\hline Haemoglobin & $\sqrt{ }$ & & & & & & & & & & & & & \\
\hline $\begin{array}{l}\text { Urine } \\
\text { lipoarabinomannan }\end{array}$ & $\sqrt{ }$ & $\sqrt{ }^{a}$ & & & & & & & & & & & & \\
\hline \multicolumn{15}{|c|}{$\begin{array}{l}\text { Sputum TB microscopy } \\
\text { and culture }\end{array}$} \\
\hline $\begin{array}{l}\text { Urine/dried blood spot } \\
\text { for storage }\end{array}$ & $\sqrt{ }$ & & & & & & & $\sqrt{ }$ & & & & & & \\
\hline $\begin{array}{l}\text { TB patient follow-up } \\
\text { visit }\end{array}$ & & & $\sqrt{ }^{\mathrm{b}}$ & & & & & & & & & & & \\
\hline Patient record review & & $\sqrt{ }$ & & & $\sqrt{ }$ & & $\sqrt{ }$ & & $\sqrt{ }$ & & & $\sqrt{ }$ & & $\sqrt{ }$ \\
\hline $\begin{array}{l}6 \text { month } \\
\text { questionnaire }^{c}\end{array}$ & & & & & & & $\sqrt{ }$ & & & & & & & $\sqrt{ }$ \\
\hline Patient contact calls & & $\sqrt{ }$ & & $\sqrt{ }$ & $\sqrt{ }$ & $\sqrt{ }$ & $\sqrt{ }$ & & $\sqrt{ }$ & & $\sqrt{ }$ & $\sqrt{ }$ & $\sqrt{ }$ & $\sqrt{ }$ \\
\hline
\end{tabular}

${ }^{\mathrm{a}}$ For those at medium probability of TB according to the study algorithm. ${ }^{\mathrm{b}}$ For those started on TB treatment, to check if stable on TB treatment and ready to start antiretroviral therapy. ${ }^{\mathrm{C}} \mathrm{A}$ subset of participants also have this questionnaire repeated at 12 months from enrolment. TB, tuberculosis. 
of Gauteng, North West and Limpopo. It is registered with the South African Medicines Control Council as a phase 4 clinical trial (identifier: N2/19/8/2 () \#20120157), and with Current Controlled Trials (identifier: ISRCTN35344604 [23]) and the South African registry (identifier: DOH-270812-3902).

The trial results will be communicated to stakeholders through dissemination meetings and to trial participants using language-appropriate information sheets. Investigators will present results at relevant conferences, and submit manuscript(s) to peer-reviewed journals. Public access to the participant-level dataset of main trial results and statistical code will be made available.

\section{Trial governance}

The trial is governed by the Trial Steering Committee (TSC) and the Data Monitoring Committee (DMC). The TSC oversees the trial, monitors its progress and receives reports from the DMC, and advises the Chief Investigator and investigator team. The DMC's role is to protect and serve trial patients, to assist and advise the Chief Investigator and the TSC so as to protect the validity and credibility of the trial and monitor the overall conduct of the clinical trial. The DMC meets approximately every six months, followed by a TSC meeting. Protocol amendments are submitted to all ethics committees and communicated to all investigators, the TSC and DMC.

\section{Discussion}

This study aims to evaluate whether early mortality can be reduced, among ambulatory people with advanced HIV disease not taking ART, by an algorithm which enables nurses in primary care clinics to identify patients at highest risk and rapidly start them on tuberculosis treatment. A number of other recent trials, summarised in Table 2, aim to address the problem of high early mortality among people with advanced HIV disease entering care. The PrOMPT [24] and REMEMBER [25] studies aimed to evaluate empirical tuberculosis treatment among people with HIV and very low CD4 counts who screened negative for active tuberculosis at study entry. Recruitment to the PrOMPT study was much slower than anticipated and as a result the study was discontinued. The REMEMBER trial, with sites in Brazil, Haiti, India, Peru and five African countries, has a primary outcome of mortality at 24 weeks. Recruitment is complete and results are expected in 2015. The STATIS trial compares, among people with no overt evidence of tuberculosis at study entry, empirical tuberculosis treatment to intensive investigation for tuberculosis among HIV-positive patients with a CD4 count under 100 cells/ $\mu \mathrm{L}$ in Cambodia, Côte d'Ivoire, Uganda and Vietnam [26]. Intensive investigation comprises Xpert MTB/RIF, urine LAM tests and a chest radiograph at baseline and at every follow-up visit, with tuberculosis treatment guided by these investigations. The primary outcome is death or invasive bacterial infection by 24 weeks.

Other studies are testing alternative interventions designed to reduce early mortality during ART. The REMSTART study included patients with a CD4 count under 200 cells $/ \mu \mathrm{L}$ in Tanzania and Zambia, who were individually randomised to standard of care or a complex intervention comprising immediate ART start, screening for cryptococcal antigen, weekly home visits from lay workers and screening with Xpert MTB/RIF at around six weeks [27]. Recruitment to REMSTART is complete. The REALITY trial is recruiting ART-naive adults and children over five years with a CD4 count under 100 cells $/ \mu \mathrm{L}$ in Kenya, Malawi, Uganda and Zimbabwe [28]. There are three interventions: a) intensification of ART using raltegravir in addition to a standard three-drug ART regimen, compared to standard ART; b) multi-drug prophylaxis against co-infections using isoniazid, pyridoxine and co-trimoxazole plus fluconazole for 12 weeks, azithromycin for five days and single-dose albendazole, compared to co-trimoxazole alone for 12 weeks followed by isoniazid and pyridoxine and c) food supplementation compared to standard of care.

We planned the TB Fast Track trial to be as pragmatic (rather than explanatory) as possible, because we wanted to know if the intervention would improve patient outcomes under routine conditions in resource-limited settings. Assessing against criteria set out by Thorpe et al. [29], features of the trial consistent with the pragmatic end of this spectrum include few exclusion criteria; an intervention delivered by nurses, as it would be if the intervention was implemented in practice; a routine practice comparator, with few study activities in the control arm likely to alter the standard of care; few study follow-up visits; a primary outcome, all-cause mortality, which is objective and clinically meaningful; no special measures to promote participant adherence and an intention-to-treat primary analysis.

In a few domains, the trial is less pragmatic: the initial study intervention (implementation of a management algorithm) is relatively inflexible and undertaken by study staff, although after the initial management pathway has been determined (treatment for tuberculosis followed by ART, or ART alone), further management is by clinic staff following their usual procedures.

In line with this pragmatic approach, we tried to minimise (in both arms of the study) additional tests for tuberculosis which are not currently routine practice in primary care clinics, which would make the intervention very difficult to replicate in resource-constrained settings. The exception to this principle was that we requested a 
Table 2 Summary of trials of interventions to reduce early mortality among HIV-positive people starting antiretroviral therapy

\begin{tabular}{|c|c|c|c|c|c|c|}
\hline Study & Setting & $\begin{array}{l}\text { Randomisation } \\
\text { level }\end{array}$ & $\begin{array}{l}\text { Study population } \\
\text { (main criteria) }\end{array}$ & Intervention & Outcome(s) & $\begin{array}{l}\text { Expected } \\
\text { date of } \\
\text { results }\end{array}$ \\
\hline $\operatorname{PrOMPT}^{\mathrm{a}}[24]$ & $\begin{array}{l}\text { Gabon, } \\
\text { Mozambique, } \\
\text { South Africa, } \\
\text { Uganda }\end{array}$ & Individual & $\begin{array}{l}\text { CD4 count }<50 \text { and body } \\
\text { mass index }<18 ; \text { no } \\
\text { previous TB treatment; } \\
\text { aged } \geq 18 \text { years; Sputum } \\
\text { smear negative; and not } \\
\text { fulfilling World Health } \\
\text { Organization criteria for } \\
\text { smear-negative TB. }\end{array}$ & $\begin{array}{l}\text { 4-drug TB treatment, } \\
\text { followed by ART within } \\
2 \text { weeks. Comparator: ART } \\
\text { alone }\end{array}$ & $\begin{array}{l}\text { Primary: all-cause mortality } \\
\text { in the first } 24 \text { weeks after } \\
\text { initiation of ART, CD4 cell } \\
\text { increase, safety, HIV viral } \\
\text { suppression, TB incidence } \\
\text { after ART initiation }\end{array}$ & $\mathrm{N} / \mathrm{A}$ \\
\hline REMEMBER [25] & $\begin{array}{l}\text { Brazil, Haiti, } \\
\text { India, Kenya, } \\
\text { Malawi, Peru, } \\
\text { South Africa, } \\
\text { Tanzania, } \\
\text { Zimbabwe }\end{array}$ & Individual & $\begin{array}{l}\text { CD4 count }<50 \text {; aged } \\
\geq 13 \text { years; Karnofsky } \geq 30 ; \\
\text { no previous TB treatment } \\
\text { (within } 96 \text { weeks). Those } \\
\text { with confirmed or } \\
\text { probable TB excluded. }\end{array}$ & $\begin{array}{l}\text { ART initiation within } 3 \text { days } \\
\text { and } 4 \text {-drug TB treatment } \\
\text { within } 7 \text { days of ART start. } \\
\text { Comparator: ART initiation } \\
\text { within } 3 \text { days }\end{array}$ & $\begin{array}{l}\text { Primary: survival at } \\
24 \text { weeks; survival over } \\
96 \text { weeks; time to AIDS } \\
\text { progression; AIDS-free } \\
\text { survival at } 24 \text { and } 28 \text { weeks; } \\
\text { HIV viral load at } 2,24 \text { and } \\
48 \text { weeks; safety }\end{array}$ & May 2016 \\
\hline REMSTART [27] & $\begin{array}{l}\text { Tanzania, } \\
\text { Zambia }\end{array}$ & Individual & $\begin{array}{l}\text { Initially CD4 count }<100 \text {, } \\
\text { broadened to }<200 \\
\text { following slow enrolment; } \\
\text { aged } \geq 18 \text { years. All } \\
\text { screened for TB at } \\
\text { enrolment with Xpert } \\
\text { MTB/RIF }\end{array}$ & $\begin{array}{l}\text { Rapid initiation of ART, } \\
\text { screening for cryptococcal } \\
\text { antigen, weekly home visits } \\
\text { for } 4 \text { weeks by lay workers } \\
\text { and rescreening for TB } \\
\text { using Xpert MTB/RIF at } \\
6 \text { weeks. Comparator: } \\
\text { standard of care }\end{array}$ & $\begin{array}{l}\text { Primary: all-cause mortality } \\
\text { at } 12 \text { months, patient } \\
\text { retention, hospital admission, } \\
\text { outpatient attendances, TB, } \\
\text { cryptococcal meningitis, ART } \\
\text { adherence }\end{array}$ & Dec 2014 \\
\hline REALITY [28] & $\begin{array}{l}\text { Kenya, } \\
\text { Malawi, } \\
\text { Uganda, } \\
\text { Zimbabwe }\end{array}$ & Individual & $\begin{array}{l}C D 4 \text { count }<100, \text { aged } \\
\geq 5 \text { years }\end{array}$ & $\begin{array}{l}2 \times 2 \times 2 \text { factorial: a) intensified } \\
\text { ART (triple therapy plus } \\
\text { raltegravir) for } 12 \text { weeks; } \\
\text { b) multidrug prophylaxis } \\
\text { against co-infections } \\
\text { (isoniazid, pyridoxine, co- } \\
\text { trimoxazole and fluconazole } \\
\text { for } 12 \text { weeks; azithromycin } \\
\text { for } 5 \text { days; single dose } \\
\text { albendazole); c) ready-to-use } \\
\text { supplementary food for } \\
12 \text { weeks. Comparator: } \\
\text { standard of care including } \\
\text { co-trimoxazole, with } \\
\text { isoniazid and pyridoxine } \\
\text { after } 12 \text { weeks }\end{array}$ & $\begin{array}{l}\text { Primary: mortality over the } \\
\text { first } 24 \text { weeks after starting } \\
\text { ART, mortality at } 48 \text { weeks } \\
\text { after starting ART, safety, } \\
\text { endpoints relating to the } \\
\text { specific mechanisms of } \\
\text { action of each intervention }\end{array}$ & Aug 2015 \\
\hline STATIS [26] & $\begin{array}{l}\text { Cambodia, } \\
\text { Côte d'Ivoire, } \\
\text { Uganda, } \\
\text { Vietnam }\end{array}$ & Individual & $\begin{array}{l}\text { CD4 count }<100 \text {; starting } \\
\text { ART; aged } \geq 18 \text { years. } \\
\text { Excluded if overt evidence } \\
\text { of TB }\end{array}$ & $\begin{array}{l}\text { Empirical treatment. } \\
\text { Comparator: extensive TB } \\
\text { screening (point of care } \\
\text { urine lipoarabinomannan, } \\
\text { sputum Xpert MTB/RIF, } \\
\text { chest radiograph for all at } \\
\text { enrolment and those with } \\
\text { TB symptoms or signs at all } \\
\text { follow-up visits) }\end{array}$ & $\begin{array}{l}\text { Primary: composite of (i) } \\
\text { 24-week all-cause mortality } \\
\text { and (ii) 24-week incidence } \\
\text { of invasive bacterial } \\
\text { infections; incidence of TB; } \\
\text { safety }\end{array}$ & Jun 2017 \\
\hline TB Fast Track [23] & South Africa & Clinic & $\begin{array}{l}\text { CD4 count } \leq 150 \text {; not on } \\
\text { ART and willing to start } \\
\text { ART; aged } \geq 18 \text { years. No } \\
\text { pre-screening for TB prior } \\
\text { to enrolment }\end{array}$ & $\begin{array}{l}\text { Management strategy to } \\
\text { identify those at highest } \\
\text { risk of TB, so that they can } \\
\text { start TB treatment } \\
\text { immediately, followed by } \\
\text { ART. Comparator: standard } \\
\text { of care }\end{array}$ & $\begin{array}{l}\text { Primary: 6-month mortality, } \\
\text { severe morbidity over } \\
6 \text { months, time to initiation } \\
\text { of ART, retention in ART } \\
\text { care, safety }\end{array}$ & Dec 2015 \\
\hline
\end{tabular}


sputum specimen, processed for smear and culture in a research laboratory, from all participants in the intervention arm in order to have a gold standard against which to assess our management algorithm. These results are delivered to clinic staff as soon as they become available. In our experience to date, they rarely change the patient's management since most patients are sputum-smear negative, and by the time the culture result is available, most patients have already started tuberculosis treatment. The disadvantage of this minimalist approach to additional diagnostic tests is that we do not have a rigorous gold standard against which to assess the performance of our algorithm. In addition, if the trial succeeds in reducing mortality, it may be difficult to assess the relative contributions of prompt tuberculosis treatment and earlier initiation of ART.

A potential challenge to the TB Fast Track trial is the evolution of South African guidelines for the management of HIV-positive patients. The trial was designed and awarded funding prior to the roll-out of Xpert MTB/RIF in South Africa. When the trial started, no trial clinics had access to Xpert MTB/RIF, but by April 2013 all trial clinic guidelines recommended Xpert $\mathrm{MTB} / \mathrm{RIF}$ as the first test for people being investigated for tuberculosis. If this guideline was followed rigorously, it could potentially increase detection of active tuberculosis in all study clinics, which could reduce the effect of the intervention. In addition, guidance concerning the initiation of ART has been modified in South Africa, such that several provinces recommend immediate (same day) ART initiation among HIV-positive people who are not taking ART and who have CD4 counts below 200 cells $/ \mu \mathrm{L}$. The guidance does not make clear how screening for active tuberculosis should fit into the process of rapid ART initiation. Rigorous implementation of this guidance would result in individuals in both arms of the study starting ART more rapidly than previously, and thus has the potential to reduce the effect of the intervention. On the other hand, all changes in routine practice which are likely to reduce early mortality are important, and the trial is most useful if it evaluates the intervention against the contemporary standard of care.

This trial will test whether a management algorithm, using tests which can be performed in primary care settings with very few resources, will enable nurses to identify HIV-positive patients at the highest risk of tuberculosis to facilitate prompt treatment, and thus reduce early mortality. The intervention will inevitably result in over-treatment for tuberculosis. We believe this can be justified in the current context where diagnostic tests for tuberculosis lack sensitivity amongst the highest risk patients, and there is consistent evidence that untreated tuberculosis is the most important cause of death in this patient group. There remains an urgent priority for better diagnostic tests for tuberculosis, encompassing those with advanced HIV disease and extrapulmonary tuberculosis, or both, which will render the approach of empirical treatment unnecessary.

\section{Trial status}

The study completed enrolment on 23 December 2014.

\begin{abstract}
Abbreviations
ART: Antiretroviral therapy; BMI: Body mass index; CXR: Chest radiograph; DALY: Disability-adjusted life year; DMC: Data monitoring committee; LAM: Lipoarabinomannan; TSC: Trial steering committee.
\end{abstract}

\section{Competing interests}

The authors declare that they have no competing interests.

\section{Authors' contributions}

AG, SC and CH conceived of the study. SC, KF, CH, GC, SJ, AV, SD and MT initiated the study design and MT led the implementation. AG is the grant holder. KF provided statistical expertise in clinical trial design and will be conducting the primary statistical analysis. All authors contributed to refinement of the study protocol and approved the final manuscript.

\section{Acknowledgements}

The trial sponsor is the London School of Hygiene \& Tropical Medicine, Keppel Street, London WC1E 7HT. The trial is funded by the Global Health Trials (UK Department for International Development/Medical Research Council/Wellcome Trust, G1100689). The autopsy substudy is funded by the Bill and Melinda Gates Foundation (OPP1083118). The funder and study sponsor have no role in the study design and will have no role in the execution of the study, analyses and interpretation of data, or decision to submit results for publication.

\section{Trial Steering Committee}

Professor Bertel Squire, Liverpool School of Tropical Medicine (Chair); Dr Lindiwe Mvusi, Director, DOTS Strategy Coordination, South African Department of Health; Bonginkosi Mthembu, Treatment Action Campaign, South Africa; Professor Harry Hausler, Director, TB/HIV Care Association, South Africa; Professor Francois Venter, Wits Reproductive Health and HIV Institute, South Africa.

\section{Data Monitoring Committee}

Professor Andrew Nunn (Chair), MRC Clinical Trials Unit, UK; Dr Kogieleum Naidoo, University of KwaZulu-Natal, South Africa; Dr Jonathan Levin, MRC Uganda, Uganda.

\section{Author details}

'Department of Infectious Disease Epidemiology, London School of Hygiene \& Tropical Medicine, Keppel Street, London WC1E 7HT, UK. ${ }^{2}$ Aurum Institute, 29 Queens Road, Johannesburg 2041, South Africa. ${ }^{3}$ School of Medicine, Johns Hopkins University, 1503 E. Jefferson Street, Baltimore, Maryland 21231, USA. ${ }^{4}$ Technical Assistance Cluster, Foundation for Professional Development, 173 Mary Road, Pretoria 0184, South Africa. ${ }^{5}$ Department of Global Health and Development, London School of Hygiene \& Tropical Medicine, Keppel Street, London WC1E 7HT, UK. ${ }^{6}$ Department of Clinical Research, London School of Hygiene \& Tropical Medicine, Keppel Street, London WC1E 7HT, UK.

Received: 21 November 2014 Accepted: 13 March 2015

Published online: 28 March 2015

\section{References}

1. Gupta A, Nadkarni G, Yang WT, Chandrasekhar A, Gupte N, Bisson GP, et al. Early mortality in adults initiating antiretroviral therapy (ART) in low- and middle-income countries (LMIC): a systematic review and meta-analysis. PLoS One. 2011;6(12):e28691. 
2. Cox JA, Lukande RL, Lucas S, Nelson AM, Van Marck E, Colebunders R. Autopsy causes of death in HIV-positive individuals in sub-Saharan Africa and correlation with clinical diagnoses. AIDS Rev. 2010;12(4):183-94.

3. Wong EB, Omar T, Setlhako GJ, Osih R, Feldman C, Murdoch DM, et al. Causes of death on antiretroviral therapy: a post-mortem study from South Africa. PLoS One. 2012;7(10):e47542.

4. Karat SC, Tlali M, Fielding K, Hoffman C, Johnson S, Churchyard G, et al. Investigation for tuberculosis delays start of antiretroviral therapy in South African primary care clinics. Int J Tuberc Lung Dis. 2013; 17(12) supplement 2:\$431

5. Churchyard GJ, Chaisson RE, Maartens G, Getahun H. Tuberculosis preventive therapy: an underutilised strategy to reduce individual risk of TB and contribute to TB control. S Afr Med J. 2014;104(5):339-43.

6. Nicol MP, Whitelaw A, Wendy S. Using Xpert MTB/RIF. Curr Respir Med Rev. 2013:9:187-92.

7. World Health Organization. Automated real-time nucleic acid amplification technology for rapid and simultaneous detection of tuberculosis and rifampicin resistance: Xpert MTB/RIF assay for the diagnosis of pulmonary and extrapulmonary TB in adults and children, Policy update. Geneva: WHO; 2013. Available from: http://www.stoptb.org/wg/gli/assets/documents/WHO \%20Policy\%20Statement\%20on\%20Xpert\%20MTB-RIF\%202013\%20pre\% 20publication\%2022102013.pdf. (Accessed 23March2015).

8. Boehme CC, Nabeta P, Hillemann D, Nicol MP, Shenai S, Krapp F, et al. Rapid molecular detection of tuberculosis and rifampin resistance. N Engl J Med. 2010;363(11):1005-15.

9. Churchyard GJ, Stevens WS, Mametja LD, McCarthy KM, Chihota V, Nicol MP, et al. Xpert MTB/RIF replacing sputum microscopy as the initial diagnostic test for tuberculosis: a cluster-randomised trial embedded in South African roll-out. Lancet Global Health. 2015. in press.

10. Theron G, Zijenah L, Chanda D, Clowes P, Rachow A, Lesosky M, et al. Feasibility, accuracy, and clinical effect of point-of-care Xpert MTB/RIF testing for tuberculosis in primary-care settings in Africa: a multicentre, randomised, controlled trial. Lancet. 2014;383(9915):424-35.

11. Lawn SD. Point-of-care detection of lipoarabinomannan (LAM) in urine for diagnosis of HIV-associated tuberculosis: a state of the art review. BMC Infect Dis. 2012;12:103.

12. Hanifa Y, Fielding KL, Charalambous S, Variava E, Luke B, Churchyard GJ, et al. Tuberculosis among adults starting antiretroviral therapy in South Africa: the need for routine case finding. Int J Tuberc Lung Dis. 2012;16(9):1252-9.

13. Kerkhoff AD, Wood R, Vogt M, Lawn SD. Predictive value of anemia for tuberculosis in HIV-infected patients in Sub-Saharan Africa: an indication for routine microbiological investigation using new rapid assays. J Acquir Immune Defic Syndr. 2014;66(1):33-40.

14. Kufa T, Mngomezulu V, Charalambous S, Hanifa Y, Fielding K, Grant AD, et al. Undiagnosed tuberculosis among HIV clinic attendees: association with antiretroviral therapy and implications for intensified case finding isoniazid preventive therapy, and infection control. J Acquir Immune Defic Syndr. 2012;60(2):e22-8.

15. Lawn SD, Brooks SV, Kranzer K, Nicol MP, Whitelaw A, Vogt M, et al. Screening for HIV-associated tuberculosis and rifampicin resistance before antiretroviral therapy using the Xpert MTB/RIF assay: a prospective study. PLoS Med. 2011;8(7):e1001067.

16. Moore D, Liechty C, Ekwaru P, Were W, Mwima G, Solberg P, et al. Prevalence, incidence and mortality associated with tuberculosis in HIV-infected patients initiating antiretroviral therapy in rural Uganda. AIDS. 2007;21(6):713-9.

17. Russell EC, Charalambous S, Pemba L, Churchyard GJ, Grant AD, Fielding K. Low haemoglobin predicts early mortality among adults starting antiretroviral therapy in an HIV care programme in South Africa: a cohort study. BMC Public Health. 2010;10:433

18. Shah M, Variava E, Holmes CB, Coppin A, Golub JE, McCallum J, et al. Diagnostic accuracy of a urine lipoarabinomannan test for tuberculosis in hospitalized patients in a high HIV prevalence setting. J Acquir Immune Defic Syndr. 2009;52(2):145-51.

19. Hayes RJ, Moulton LH. Cluster randomised trials. Boca Raton, FL: Chapman \& Hall/CRC Press; 2009

20. Simms V, Matiku S, Ngowi B, Chanda D, Lesikari S, Bottomley C, et al. Integrating public health research trials into health systems in Africa: individual or cluster randomisation? Tropical Med Int Health. 2014:19(1):123-7.
21. Hoffmann CJ, Fielding KL, Johnston V, Charalambous S, Innes C, Moore RD, et al. Changing predictors of mortality over time from CART start: implications for care. J Acquir Immune Defic Syndr. 2011;58(3):269-76.

22. Hoffmann CJ, Lewis JJ, Dowdy DW, Fielding KL, Grant AD, Martinson NA et al. Mortality associated with delays between clinic entry and ART initiation in resource-limited settings: results of a transition-state model. J Acquir Immune Defic Syndr. 2013;63(1):105-11.

23. Grant AD. TB Fast Track trial. Available from: http://www.isrctn.com/ ISRCTN35344604. Accessed 23 March 2015.

24. Cobelens F, Manabe Y. Prevention of Early Mortality by Presumptive Tuberculosis (TB) Treatment (PrOMPT). Available from: http://clinicaltrials. gov/ct2/show/study/NCT01417988. Accessed 23 March 2015.

25. Hosseinipour MC, Kumwenda J. REMEMBER: Reducing Early Mortality \& Morbidity by Empiric Tuberculosis (TB) Treatment. Available from: http:// clinicaltrials.gov/ct2/show/NCT01380080. Accessed 23 March.

26. Blanc FX, Domoua KMS. Systematic Empirical vs. Test-guided Anti-TB Treatment Impact in Severely Immunosuppressed HIV-infected Adults Initiating ART with CD4 Cell Counts $<100 / \mathrm{mm}^{3}$ (STATIS trial). Available from: http:// clinicaltrials.gov/ct2/show/study/NCT02057796. Accessed 23 March 2015.

27. Egwaga S. Home care and routine: cryptococcal meningitis screening among patients starting antiretroviral therapy with advanced disease (REMSTART trial). Available from: http://www.isrctn.com/ISRCTN20410413. Accessed 23 March 2015

28. Gibb D. Reduction of Early mortALITY in HIV-infected African adults and children starting antiretroviral therapy: REALITY trial. Available from: http:// www.isrctn.com/ISRCTN43622374. Accessed 23 March 2015.

29. Thorpe KE, Zwarenstein M, Oxman AD, Treweek S, Furberg CD, Altman DG, et al. A pragmatic-explanatory continuum indicator summary (PRECIS): a tool to help trial designers. J Clin Epidemiol. 2009;62(5):464-75.

\section{Submit your next manuscript to BioMed Central and take full advantage of:}

- Convenient online submission

- Thorough peer review

- No space constraints or color figure charges

- Immediate publication on acceptance

- Inclusion in PubMed, CAS, Scopus and Google Scholar

- Research which is freely available for redistribution 